\title{
Existence and Smoothness of Solution of Navier-Stokes Equation on $R^{3}$
}

\author{
Ognjen Vukovic \\ Department for Finance, University of Liechtenstein, Vaduz, Liechtenstein \\ Email: ognjen.vukovic@uni.li, oggyvukovich@gmail.com
}

Received 13 May 2015; accepted 12 June 2015; published 15 June 2015

Copyright (C) 2015 by author and Scientific Research Publishing Inc. This work is licensed under the Creative Commons Attribution International License (CC BY). http://creativecommons.org/licenses/by/4.0/

(c) (i) Open Access

\begin{abstract}
Navier-Stokes equation has for a long time been considered as one of the greatest unsolved problems in three and more dimensions. This paper proposes a solution to the aforementioned equation on $R^{3}$. It introduces results from the previous literature and it proves the existence and uniqueness of smooth solution. Firstly, the concept of turbulent solution is defined. It is proved that turbulent solutions become strong solutions after some time in Navier-Stokes set of equations. However, in order to define the turbulent solution, the decay or blow-up time of solution must be examined. Differential inequality is defined and it is proved that solution of Navier-Stokes equation exists in a finite time although it exhibits blow-up solutions. The equation is introduced that establishes the distance between the strong solutions of Navier-Stokes equation and heat equation. As it is demonstrated, as the time goes to infinity, the distance decreases to zero and the solution of heat equation is identical to the solution of $\mathrm{N}-\mathrm{S}$ equation. As the solution of heat equation is defined in the heat-sphere, after its analysis, it is proved that as the time goes to infinity, solution converges to the stationary state. The solution has a finite $\tau$ time and it exists when $\tau \rightarrow \infty$ that implies that it exists and it is periodic. The aforementioned statement proves the existence and smoothness of solution of Navier-Stokes equation on $R^{3}$ and represents a major breakthrough in fluid dynamics and turbulence analysis.
\end{abstract}

\section{Keywords}

Navier-Stokes Equation, Millennium Problem, Nonlinear Dynamics, Fluid, Physics

\section{Introduction}

In this paper, the following form of Navier-Stokes equations in $R^{3}$ is studied:

$$
\frac{\partial}{\partial t} u_{i}+\sum_{j=1}^{n} u_{j} \cdot y \frac{\partial u_{i}}{\partial x_{j}}=v \Delta u_{i}-\frac{\partial p}{\partial x_{i}}+f_{i}(x, t)\left(x \in R^{n}, t \geq 0\right)
$$




$$
\operatorname{div} u=\sum_{i=1}^{n} \frac{\partial u_{i}}{\partial x_{i}}=0 \quad\left(x \in R^{n}, t \geq 0\right)
$$

With initial conditions

$$
u(x, 0)=u^{0}(x)\left(x \in R^{n}\right)
$$

Here $u^{0}=x, C^{\infty}$ (divergence-free vector field on $R^{n}$ ), $f_{i}(x, t)$ are the components of a given, externally applied force, $v$ is a positive coefficient (the viscosity) and $\Delta=\sum_{i=1}^{n} \frac{\partial^{2}}{\partial x_{i}^{2}}$ is the Laplacian in space variables. If Euler equations are considered, then the same set of equation must be applied with the condition that viscosity is equal to zero.

The following conditions must be satisfied as it is wanted to make sure that $u(x, t)$ does not grow large as $|x| \rightarrow \infty$ :

$$
\left|\partial_{x}^{\alpha} u^{o}(x)\right| \leq C_{\alpha K}(1+|x|)^{-K} \text { on } R^{n} \text { for any } \alpha \text { and } K
$$

And

$$
\left|\partial_{x}^{\alpha} \partial_{t}^{m} f(x, t)\right| \leq C_{\alpha m K}(1+|x|+t)^{-K} \text { on } R^{n} x[0, \infty[\text { for any } \alpha, m, K
$$

The accepted solution of N-S is physically reasonable if it only satisfies:

$$
p, u \in C^{\infty}\left(R^{n} x[0, \infty[)\right.
$$

And

$$
\int_{R^{n}}|u(x, t)| \mathrm{d} x<C \text { for all } t \geq 0 \text { (bounded energy) }
$$

At the same time, it is possible to look at spatially periodic solutions. We can assume the following conditions:

$$
u^{o}\left(x+e_{j}\right)=u^{o}(x), f\left(x+e_{j}, t\right)=f(x, t) \text { for } 1 \leq j \leq n
$$

Under the condition that $e_{j}=j^{\text {th }}$ is unit vector in $R^{n}$. It must be assumed that $u^{o}$ is smooth and that

$$
\left|\partial_{x}^{\alpha} \partial_{t}^{m} f(x, t)\right| \leq C_{\alpha m K}(1+t)^{-K} \text { on } R^{3} x[0, \infty[\text { for any } \alpha, m, K
$$

The solution is then accepted if it satisfies:

$$
u(x, t)=u\left(x+e_{j}, t\right) \text { on } R^{3} x[0, \infty[\text { for } 1 \leq j \leq n
$$

And

$$
p, u \in C^{\infty}\left(R^{n} x[0, \infty[)\right.
$$

The problem is to find and analyze whether a strong, physically reasonable solution exists for the NavierStokes equation.

The statement that will be proved is existence and smoothness of Navier-Stokes solutions on $R^{3}$. Take $v>$ 0 and $n=3$. Let $u^{o}(x)$ be any smooth, divergence-free vector field satisfying (1.4). Take $f(x, t)$ to be identically zero. Then there exist smooth functions $p(x, t), u_{i}(x, t)$ on $R^{3} x[0, \infty]$ and the above conditions and equations are satisfied.

\section{Results}

Firstly, the definition of turbulent solutions (Oliver and Titti) [1] is provided. We must define the set of all $C^{\infty}$ real vector functions $\varphi$ with compact support in $R^{n}$ such that $\operatorname{div} \varphi=0$. We define $L_{\sigma}^{r}$ as the closure of 
$C_{0, \sigma}^{\infty}$ with respect to $L^{r}$ norm \|\|$_{I_{r}} ;(.,$.$) is the inner product in L^{2} . L^{r}$ stands for the usual $L^{r}$-space over $R^{n}$, $1 \leq r \leq \infty . H_{0, \sigma}^{1}$ is the closure of $C_{0, \sigma}^{\infty}$ with respect to the norm $\|\phi\|_{H^{1}}=\|\phi\|_{2}+\|\nabla \phi\|_{2}$ where $\nabla \varphi=\left(\frac{\partial \varphi_{i}}{\partial x_{j}}\right)_{i, j=1, \cdots, n}$. When $X$ is a Banach space, \|\|$_{X}$ denotes the norm on X. $C^{m}\left(\left[t_{1}, t_{2}\right] ; X\right)$ and $L^{r}\left(\left[t_{1}, t_{2}\right] ; X\right)$ are the Banach spaces, where $m=0,1, \cdots$, and $t_{1}$ and $t_{2}$ are real numbers such that $t_{1}<t_{2} . C$ denotes various constants.

Def 1. (Oliver and Titti) [1] A turbulent solution of Navier-Stokes equation is defined as following:

$$
\text { 1) } u \in L^{\infty}\left(0, \infty ; L_{\sigma}^{2}\right) \cap L^{2}\left(0, T ; H_{0, \sigma}^{1}\right) \text { for all } 0<T<\infty
$$

The relation

$$
\text { 2) } \int_{0}^{T}[-(u, \partial \varphi / \partial t)+(\nabla u, \nabla \varphi)+(u \cdot \nabla u, \varphi)] \mathrm{d} t=(a, \varphi(0))
$$

Holds for almost all $T$ and all $\varphi \in C^{1}\left(\left[0, T\left[; H_{0, \sigma}^{1} \cap L^{n}\right)\right.\right.$ such that $\varphi(\cdot, T)=0$

Strong energy inequality

$$
\text { 3) }\|u(t)\|_{2}^{2}+2 \int_{s}^{t}\|\nabla u(\tau)\|_{2}^{2} \mathrm{~d} \tau \leq\|u(s)\|_{2}^{2}
$$

Holds for almost all $s \geq 0$ including $s=0$, and all $t>s$.

It is necessary to introduce the Stokes operator $A_{r}$ in $L_{\sigma}^{r}$. The following Helmholtz decomposition is obtained:

$$
L^{r}=L_{\sigma}^{r} \otimes G^{r}, 1<r<\infty
$$

where $G^{r}=\left\{\nabla p \in L^{r} ; p \in L_{\text {loc }}^{r}\right\}$. $P_{\sigma}$ denotes the projection from $L^{r}$ onto $L_{\sigma}^{r}$. A defines the Stokes operator with domain $D\left(A_{r}\right)=H^{2, r} \cap L_{\sigma}^{r}$. A denotes the Stokes operator $A_{r} .\left\{E_{\lambda}\right\}_{\lambda} \geq 0$ denotes the spectral decomposition of self-adjoint operator A.

The existence of turbulent solutions for $n=3$ and $n=4$ is given by Leray and Kato. In order to derive the next results, theorem from Takahiro Okabe will be introduced.

Theorem 1. Let $2 \leq n \leq 4$ and let $r>1$ and $m \geq 0$ be

For $n=2$,

$$
1<r<4 / 3,0 \leq m<4 / r-3 \text { and }
$$

For $n=3,4$

$$
1<r<\frac{n}{n-1}, 0 \leq m<\frac{n}{r}-(n-1)
$$

Suppose that $K_{m, \alpha}^{\delta}$ for $K_{m, \alpha}^{\delta}=\left\{\varphi \in L^{2} ; \mid \hat{\varphi}\left(\left.\xi|\geq \alpha| \xi\right|^{m}\right.\right.$ for $\left.|\xi| \leq \delta\right\}$ for $\alpha, \delta>0$ and $m \geq 0$. If $a \in L_{\sigma}^{r} \cap L_{\sigma}^{2} \cap K_{m, \alpha}^{\delta, \alpha}$ for some $\alpha, \delta>0$ then for every turbulent solution $u(t)$ there exist $T>0$ and $C(n, r, m, \delta, \alpha, a)>0$ such that:

$$
\left|\frac{\left\|E_{\lambda} u(t)\right\|}{\|u(t)\|_{2}}-1\right| \leq \frac{C}{\lambda} t^{-(n / r-n+1-m)}
$$

holds for all $\lambda$ and for all $t>T$

Def 2. Let $n<r<\infty, a \in L_{\sigma}^{n}$. A measurable function $u$ defined on $R^{n} \times(0, \infty)$ is called a global strong solution of Navier-Stokes equation if:

$$
u \in C\left([0, \infty) ; L_{\sigma}^{n}\right) \cap C\left((0, \infty) ; L^{r}\right)
$$

$\frac{\partial u}{\partial t}, A u \in C\left((0, \infty) ; L_{\sigma}^{n}\right)$ and $u$ satisfies: 


$$
\frac{\partial u}{\partial t}+A u+P_{\sigma}(u \cdot \nabla u)=0, t>0
$$

where $P_{\sigma}(u \cdot \nabla u)$ denotes the projection from $L^{r}$ onto $L_{\sigma}^{r}$ of the product of the divergence of solution $u$ and the solution itself.

Takahiro Okabe [2], in his paper named "Asymptotic energy concentration in the phase of the weak solutions to the Navier-Stokes equation", proves that turbulent solutions of Navier-Stokes equation become strong solutions after some definite time. So for the turbulent solution of $u(t)$ of Navier-Stokes equation there exists $T_{*}>0$ such that $u(t)$ is a strong solution of Navier-Stokes equation on $\left[T_{*}, \infty\right)$, then the energy identity exists:

$$
\frac{\mathrm{d}}{\mathrm{d} t}\|u(t)\|_{2}^{2}+2\left\|A^{1 / 2} u(t)\right\|_{2}^{2}=0
$$

For $t \geq T_{*}$. For any fixed $\lambda>0$, the second term in (16) is estimated from below as:

$$
\left\|A^{1 / 2} u(t)\right\|_{2}^{2}=\int_{0}^{\infty} \rho \mathrm{d}\left[E_{p} u\right]_{2}^{2} \geq \int_{\lambda}^{\infty} \rho \mathrm{d}\left[E_{p} u\right]_{2}^{2} \geq \lambda \int_{\lambda}^{\infty} \mathrm{d}\left[E_{p} u\right]_{2}^{2} \geq \frac{\lambda}{2}\left(\|u(t)\|_{2}^{2}-\left\|E_{\lambda} u(t)\right\|_{2}^{2}\right)
$$

From (16) to (18), the following is obtained:

$$
\frac{\mathrm{d}}{\mathrm{d} t}\|u(t)\|_{2}^{2}+\lambda\|u(t)\|_{2}^{2} \leq \lambda\left\|E_{\chi} u(t)\right\|_{2}^{2}
$$

Afted dividing the both sides of (19) by $\lambda\|u(t)\|_{2}^{2}$, the following is obtained:

$$
\frac{\frac{\mathrm{d}}{\mathrm{d} t}\|u(t)\|_{2}^{2}}{\lambda\|u(t)\|_{2}^{2}}+1 \leq \frac{\left\|E_{\chi} u(t)\right\|_{2}^{2}}{\|u(t)\|_{2}^{2}}
$$
that:

By (17), the following is obtained $(\mathrm{d} / \mathrm{d} t)\|u(t)\|_{2}^{2}=-2\left\|A^{1 / 2} u(t)\right\|_{2}^{2}=-2\|\nabla u(t)\|_{2}^{2}$ it follows from (17) to (20)

$$
1-\frac{\left\|E_{\chi} u(t)\right\|_{2}^{2}}{\|u(t)\|_{2}^{2}} \leq \frac{2}{\lambda} \frac{\|\nabla u(t)\|_{2}^{2}}{\|u(t)\|_{2}^{2}}
$$

By introducing the new theorem that is proved in Takahiro Okabe's paper [2], the following is obtained.

Theorem 2. Let $2 \leq n \leq 4$. Let $r$ and $m$ be as

1) $n=2$

$$
1<r<\frac{4}{3}, 0 \leq m<\frac{4}{r}-3
$$

2) $n \geq 3$

$$
1<r<\frac{n}{n-1}, 0 \leq m<\frac{n}{r}-(n-1)
$$

If $a \in L_{\sigma}^{r} \cap L_{\sigma}^{2} \cap K_{0, \sigma}^{\delta}$, every turbulent solution of $u(t)$ of Navier-Stokes equation satisfies:

$$
\frac{\|\nabla u(t)\|_{2}^{2}}{\|u(t)\|_{2}^{2}} \leq O\left(t^{-(n / r-n+1-m)}\right)
$$

As $t \rightarrow \infty$.

The following theorem can be proved by using well-known Leray's structure theorem, every turbulent solution of N-S becomes the strong solution after some time. Although Kato proves that the strong solution decays in the same way as the Stokes flow $\mathrm{e}^{-t A}$, we apply different approach by using Oliver and Titti's paper [1] named "Remark on the Rate of Decay of Higher Order Derivatives for solution to the Navier-Stokes equation". 
By introducing the above mentioned theorem, the following result is obtained and it proves Theorem 1.

$$
\left|\frac{\left\|E_{\lambda} u(t)\right\|_{2}^{2}}{\|u(t)\|_{2}^{2}}-1\right| \leq \frac{C}{\lambda} t^{-(n / r-n+1-m)} \text { for all } t \geq T
$$

This result proves that energy of the molecules of fluid moving is smaller than some value determined by $C, n$, $r, m$ and it proves asymptotic energy concentration. In order to prove that turbulent solutions are at the same time strong solutions, blow-up time of solutions must be analyzed.

It is demonstrated that Navier-Stokes equation enter some class as it was already proved $D\left(\mathrm{e}^{\tau A} ; H^{r}\right)$ in arbitrarily short time. Foias and Temam have proved the following solution in the case of periodic boundary condition and for the case of the Navier-Stokes equation on the two-dimensional. Kukavica and Grujic have obtained the given results in $L^{p}$ spaces. The following lemma must be introduced and it is proved in Oliver and Titi's paper [1]:

Theorem 3. Let $\tau \geq 0, r>n / 2$ and $s<n / 2$. Then there exists a constant $C=C(n, r, s)$ such that any two functions $v$ and $w$ in $D\left(\mathrm{e}^{\tau A} ; H^{r}\right)$ satisfy the inequality:

$$
\left\|A^{r} \mathrm{e}^{\tau A}(v w)\right\|_{L^{2}} \leq C(n, r, s)\left(\left\|A^{r} \mathrm{e}^{\tau A} w\right\|_{H^{r-s}}+\left\|A^{s} \mathrm{e}^{\tau A} v\right\|_{H^{r-s}}\left\|A^{r} \mathrm{e}^{\tau A} w\right\|_{L^{2}}\right)
$$

The theorem is proved by using Plancherel theorem, the triangle inequality, the inequality

$(x+y)^{r} \leq 2^{r-1}\left(x^{r}+y^{r}\right)$ and the convolution estimate $\|f * g\|_{L^{2}} \leq\|f\|_{L^{1}}\|g\|_{L^{2}}$. These are the tools used to prove the aforementioned theorem. For further details, look at the aforementioned paper. This theorem demonstrates that the blow-up time is infinite so that the solution is existent. In order to find a solution, it must be captured in some sort of space where the function oscillates. In order to introduce the following solution, a few more results will be introduced.

Firstly, we assume the existence of solutions $u \in L^{\infty}\left([0, T] ; H^{r}\left(R^{n}\right)\right), r>n / 2$ is known for some $T>0$. In order to simplify the notation, the following is set:

$$
\begin{gathered}
J_{r}=\left\|A^{r} u\right\|_{L^{2}}^{2} \\
G_{r}=\left\|A^{r} \mathrm{e}^{\tau A} u\right\|_{L^{2}}^{2}
\end{gathered}
$$

where $\tau=\tau(t)$ is to be specified later.

Then the Gevrey norm is used to find the following result:

$$
\frac{1}{2} \dot{G}_{r}=\dot{\tau} G_{r+1 / 2}-v G_{r+1}-\int_{R^{n}} A^{r} \mathrm{e}^{\tau A}(u \cdot \nabla u) A^{r} \mathrm{e}^{\tau A} u \mathrm{~d} x
$$

The contribution of pressure term is zero because $A$ commutes with the Leray projection onto divergence free vector fields. Note that:

$$
\left\|A^{s} \mathrm{e}^{\tau A} u\right\|_{H^{r-s}} \leq c\left(G_{r}^{1 / 2}+G_{s}^{1 / 2}\right)
$$

By using Theorem 3 and Cauchy-Schwarz inequality, the following result is obtained.

$$
\left|\int_{R^{n}} A^{r} \mathrm{e}^{\tau A}(u \cdot \nabla u) A^{r} \mathrm{e}^{\tau A} u \mathrm{~d} x\right| \leq\left\|A^{r} \mathrm{e}^{\tau A}(u \cdot \nabla u)\right\|\left\|A^{r} \mathrm{e}^{\tau A} u\right\| \leq c_{1}\left(G_{s}^{1 / 2}+G_{r}^{1 / 2}\right) G_{r+1}^{1 / 2} G_{r}^{1 / 2}+c_{1}\left(G_{s+1}^{1 / 2}+G_{r+!}^{1 / 2}\right) G_{r}
$$

In order to proceed, we introduce the Theorem 4.

Theorem 4. For all nonnegative $p, q$ and $\tau$ we have the following:

$$
\left\|A^{p} \mathrm{e}^{\tau A} u\right\|^{2} \leq \mathrm{e}\left\|A^{p} u\right\|^{2}+(2 \tau)^{2 q}\left\|A^{p+q} \mathrm{e}^{\tau A} u\right\|^{2}
$$

The proof is similar to that in Theorem 3, just it should be noted that for every $x \geq 0, m>0$ one has $\mathrm{e}^{x}<\mathrm{e}+x^{m} \mathrm{e}^{x}$ since $\mathrm{e}^{x}<\mathrm{e}$ on $[0,1]$ and $\mathrm{e}^{x} \leq x^{m} \mathrm{e}^{x}$ for $x \geq 1$.

After introducing the theorem and interpolating $G_{s}$ by using Theorem 3 and Theorem 4 with $p=s$, $q=r-s$, the similar thing is done with $G_{s=1}$. If we apply the Young inequality, the following result is obtained. 


$$
\left|\int_{R^{n}} A^{r} \mathrm{e}^{\tau A}(u \cdot \nabla u) A^{r} \mathrm{e}^{\tau A} u \mathrm{~d} x\right| \leq\left\|A^{r} \mathrm{e}^{\tau A}(u \cdot \nabla u)\right\|\left\|A^{r} \mathrm{e}^{\tau A}\right\| \leq c_{1} J_{s}^{1 / 2} G_{r}^{1 / 2} G_{r+1}^{1 / 2}+c_{3} J_{s+1}^{1 / 2} G_{r}+c_{4}\left(1+\tau^{r-s}\right) G_{r} G_{r+1}^{1 / 2}
$$

where $n / 4<r / 2 \leq s<n / 2$. After setting $\tau=t$, after interpolating the first term on (26) then use the estimate on (30), the following equation is obtained:

$$
\dot{G}_{r} \leq c\left(\|u\|_{H^{1}}\right) G_{r}+c(r, s, t) G_{r}^{2}
$$

This proves that there exists a $\sigma \in(0, T]$ such that $G_{r}(0)=\left\|u_{0}\right\|_{H^{r}}^{2}$ is finite for $t \in[0, \sigma)$. This proves that if space is finite, then Garvey space is finite which demonstrates the existence of stationary solution.

Now the result of differential inequality for longer time will be derived. The radius of uniform analyticity $\rho=\tau / \sqrt{n}$ increases like $\sqrt{t}$ as $t \rightarrow \infty$ as the solutions for heat equation. First the optimal decay rate for Gevrey norm is established, the optimal decay rates for norms of finite order derivatives will be established and it will be extended to infinite order.

If first two terms of Equation (26) are considered and it is assumed that only contribution from linear terms is included, interpolation can be used as well as Young inequality while breaking the second term in several fractions. Theorem 3 provides the following:

$$
\frac{G_{r}-2 J_{r}}{2 \tau^{2}} \leq G_{r+1}
$$

we all together obtain:

$$
\begin{aligned}
& \dot{\tau} G_{r+1 / 2}-v G_{r+1} \leq \frac{1}{2} \frac{\dot{\tau}}{\tau} G_{r}+\frac{1}{2} \tau \dot{\tau} G_{r+1}-\frac{v}{2} G_{r+1}-\frac{v}{2} \frac{G_{r}-2 J_{r}}{2 \tau^{2}} \\
& =\left(\frac{1}{2} \frac{\dot{\tau}}{\tau}-\frac{v}{8} \frac{1}{\tau^{2}}\right) G_{r}+\left(\frac{1}{2} \tau \dot{\tau}-\frac{v}{8}\right) G_{r+1}-\frac{v}{8} \frac{1}{\tau^{2}} G_{r}+\frac{v}{2} \frac{1}{\tau^{2}} J_{r}-\frac{3 v}{8} G_{r+1}
\end{aligned}
$$

New theorem is introduced, it is already proved by using Plancherel theorem:

Theorem 5. Provided that $2 q \geq p \geq 0$ and $\tau>0$, the following is obtained:

$$
\left\|A^{q} u\right\|^{2} \leq c(p, q) \tau^{p-2 q}\|u\|\left\|A^{p} \mathrm{e}^{\tau A} u\right\|
$$

Combining Theorem 5 with $q=r$ and the Young inequality, the following is obtained.

$$
J_{r} \leq c_{3} \frac{1}{\tau^{2 r}} J_{0}+\frac{1}{8} G_{r}
$$

If we set $\tau=\sqrt{\left(\tau_{0}^{2}+\alpha t\right)}$ where $\tau_{0}>0$ and $0<\alpha \leq v / 2$. The following is immediately found.

$$
\frac{1}{2} \tau \dot{\tau}=\frac{\alpha}{4} \leq \frac{v}{8}
$$

So that the first two terms on the right of equation (33) are nonpositive and can be neglected. The main task is now to analyze the nonlinear terms and if possible prove that these nonlinear solutions do not affect the decay properties of the solution to infinite order. Applying the estimate on nonlinear term and by interpolating $J_{s}$ by using theorem $p=r, q=s ; J_{s}$ is interpolated in an analogous manner. By application of Young inequality, the following is found.

$$
\begin{aligned}
& \left|\int_{R^{n}} A^{r} \mathrm{e}^{\tau A}(u \cdot \nabla u) A^{r} \mathrm{e}^{\tau A} u \mathrm{~d} x\right| \\
& \leq c_{5} \tau^{(r / 2)-s} J_{0}^{1 / 4} G_{r}^{3 / 4} G_{r+1}^{1 / 2}+c_{6} \tau^{(r / 2)-s-1} J_{0}^{1 / 4} G_{r}^{5 / 4}+c_{4}\left(1+\tau^{r-s}\right) G_{r} G_{r+1}^{1 / 2} \leq c_{7} \tau^{r-2 s} J_{0}^{1 / 2} G_{r}^{3 / 2} \\
& \quad+c_{6} \tau^{(r / 2)-s-1} J_{0}^{1 / 4} G_{r}^{5 / 4}+C_{8}\left(1+\tau^{2(r-s)}\right) G_{r}^{2}+\frac{3 v}{8} G_{r+1}
\end{aligned}
$$

\section{Theoretical Findings}

The following differential inequality is obtained. 


$$
\dot{G}_{r} \leq-\frac{v}{8} \frac{1}{\tau^{2}} G_{r}+c_{3} \frac{v}{\tau^{2(r+1)}} J_{0}+2 c_{7} \tau^{r-2 s} J_{0}^{1 / 2} G_{r}^{3 / 2}+2 c_{6} \tau^{(r / 2)-s-1} J_{0}^{1 / 4} G_{r}^{5 / 4}+2 c_{8}\left(1+\tau^{2(r-s)}\right) G_{r}^{2}
$$

As we are considering global asymptotics and blow-up profiles, they are only possible in the presence of a critical controlled quantity or the combination of a subcritical and a supercritical controlled quantity. It turns out that the Navier-Stokes equation according to differential inequality tends to contract these quantities, in that way leading to a useful way to force finite time blow-up. The idea of using minimal surface area as controlled quantities originates from Hamilton. In order to discuss the blow-up time, we introduce the following well known proposition:

Assume that $\pi_{2}\left(M_{t}\right)$ is non-trivial. Let $\beta: S^{2} \rightarrow M_{t}$ be any immersed sphere not homotopic to a point. Each such sphere has an energy $E(\beta, t):=\frac{1}{2} \int_{s^{2}}|\mathrm{~d} \beta|^{2}$ using the metric $g_{t}$ at time $t$. If we define $W_{2}(t)$ to be the infimum of $E(\beta, t)$ over all such $\beta$. It turns out from standard Sacks-Uhlenbeck minimal surface theory that this infimum is actually attained. The differential inequality is obtained using structure of minimal surfaces and the Gauss-Bonnet formula [3]:

$$
\partial_{t} W_{2}(t) \leq-4 \pi-\frac{1}{2} R_{\min }(t) W_{2}(t)
$$

where $R_{\min }$ is the Ricci scalar. It demonstrates that the change of infimum of energy becomes negative in finite which is absurd. Therefore this forces blow-up in finite time. This means that the solution blows up in a finite time, which is why the surgery approach will be used.

If the above mentioned state holds, then the differential inequality, in order to make nonlinear terms of lower order, has to satisfy the following form:

$$
\frac{v}{32} \frac{1}{\tau^{2}}>c_{7} \tau^{r-2 s} J_{0}^{1 / 2} G_{r}^{1 / 2}+c_{6} \tau^{(r / 2)-s-1} J_{0}^{1 / 4} G_{r}^{1 / 4}+c_{8}\left(1+\tau^{2(r-s)}\right) G_{r}
$$

where $s \in[(r / 2),(r / 2)+1]$ is fixed. First it must be noted that $G_{r}$ is an increasing function of $\tau$, so that at the beginning at the initial time $t=0, G_{r}$ is bounded between $\left\|A^{r} u_{0}\right\|^{2}$ when $\tau=\tau_{0}=0$ and $\left\|A^{r} \mathrm{e}^{\sigma A} u_{0}\right\|^{2}$ when $\tau=\tau_{0}=\sigma$. Thus the left side of equation (39) diverges faster than the right side as $\tau \rightarrow 0$, so that we can satisfy condition at $t=0$ by choosing $\tau_{0} \in(0, \sigma]$ small enough. However, what happens when $\tau$ doesn't converge to 0 . Imagine $\tau \rightarrow \infty$, then the left part of equation is 0 and the right part is higher than zero, but that is not possible, because it is proved above that the infimum of energy becomes negative, that is absurd. So the solution must blow up in some definite and the equation must hold even for $\tau$ as a solution. This proves that the solution is existent and smooth. In order to proceed, we will analyze the nonlinear terms. After having proved that the above equation must hold even for some $\tau$ that does not converge to 0 , the only equation that must be solved is the following:

$$
\dot{G}_{r} \leq-\frac{\delta}{\tau^{2}} G_{r}+\frac{C_{4}}{\tau^{2(r+1)}} J_{0}
$$

where $\delta=v / 16$. According to assumption that there exist positive real numbers $M_{1}$ and $\gamma$ which may depend on $u_{0}$ such that $\|u(t)\|^{2} \leq \frac{M_{1}}{(1+t)^{\gamma}}$ for all $t \geq 0$ where $u(t)$ is a solution to the Navier-Stokes equation $J_{0} \leq M_{1}\left(\tau_{0} / \tau\right)^{2 \gamma}$ provided $\tau=\sqrt{\left(\tau_{0}^{2}+\alpha t\right)}$ and $J_{0}=\|u\|_{L^{2}}^{2}$, where $\tau_{0}>0$ and $0<\alpha \leq v / 2$, a final form of differential inequality is obtained.

$$
\dot{G}_{r} \leq-\frac{\delta}{\tau^{2}} G_{r}+\frac{k}{\tau^{2(\gamma+r+1)}}
$$

The integrating factor for linear differential inequality is:

$$
\exp \left(\delta \int_{0}^{t} \frac{1}{\tau_{0}^{2}+\alpha t^{\prime}} \mathrm{d} t^{\prime}\right)=\left(\frac{\tau_{0}^{2}+\alpha t}{\tau_{0}^{2}}\right)^{\delta / \alpha}
$$


So the following is obtained.

$$
\frac{\mathrm{d}}{\mathrm{d} t}\left(\tau^{2 \delta / \alpha} G_{r}\right) \leq k \tau^{2(\delta / \alpha-\gamma-r-1)}
$$

If we fix $\alpha$ small enough so that $\delta>\alpha(\gamma+r)$, the following is concluded:

$$
G_{r}(t) \leq\left(G_{r}(0)-\frac{k}{\delta-\alpha(\gamma+r)} \frac{1}{\tau_{0}^{2(\gamma+r)}}\right)\left(\frac{\tau_{0}^{2}}{\tau^{2}}\right)^{\delta / \alpha}+\frac{k}{\delta-\alpha(\gamma+r)} \frac{1}{\tau^{2(\gamma+r)}}
$$

If the condition (39) is satisfied for all $t$, estimate (44) will be global in time. It is sufficient to show the following:

$$
\frac{32}{v} \tau^{2}\left(c_{7} \tau^{r-2 s} J_{0}^{1 / 2} G_{r}^{1 / 2}+c_{6} \tau^{(1 / 2)-s-1} J_{0}^{1 / 4} G_{r}^{1 / 4}+c_{8}\left(1+\tau^{2(r-s)}\right) G_{r}\right) \leq g(t)
$$

for some non-increasing function $g(t)$. Estimate (44) shows that this is the case whenever $\gamma>0$ and

$$
G_{r}(0)>\frac{k}{\delta-\alpha(\gamma+r)} \frac{1}{\tau_{0}^{2(\gamma+r)}}
$$

which satisfies the above mentioned conditions and it proves the existence of a solution. As $\tau \rightarrow \infty$, $G_{r}$ converges to zero therefore the solution is existent at the beginning, and if the equations exist, then the solution exists in the time $\tau$.

It is obtained that:

$$
G_{r}(t) \leq \frac{c_{6}}{\tau^{2(\gamma+r)}}+O\left(\tau^{-2 \delta / \alpha}\right)
$$

The upper bound of decay is calculated and given below:

$$
\begin{aligned}
\left\|A^{m} u\right\|^{2} & \leq c(m, r) \tau^{r-2 m} J_{0}^{1 / 2} G_{r}^{1 / 2} \leq c(m, r) \tau^{r-2 m} M^{1 / 2}\left(\frac{\tau_{0}}{\tau}\right)^{\gamma}\left(\frac{c_{6}}{\tau^{2(\gamma+r)}}+O\left(\tau^{-2 \delta / \alpha}\right)\right)^{1 / 2} \\
& \leq c_{9} c(m, r) \frac{1}{\tau^{2(\gamma+m)}}\left(1+O\left(\tau^{\gamma+r-\delta / \alpha}\right)\right)
\end{aligned}
$$

where $c(m, r)$ is given above according to the following definition and maximum is attained at $|\zeta|=(2 q-p) / \tau$ so the following definition demonstrates:

$$
\begin{gathered}
c(q, p)=(2 q-p)^{2 q-p} \mathrm{e}^{-(2 q-p)} \text { for } 2 q>p \\
c(q, p)=1, \text { for } 2 q=p
\end{gathered}
$$

This proves that solution is existent even when $\tau$ does not converge to 0 .

Now in order to proceed and analyze the blow-up time, $v$ as the solution of the heat equation will be introduced. It should be proved that the solution $w=u-v$ between Navier-Stokes and heat solution in $\left\|A^{m}.\right\|$ can be made sufficiently small so that $u$ must decay at the same rate.

First an estimate on the difference $w$ in $D\left(\mathrm{e}^{\tau A} ; H^{r}\right)$. Clearly, it satisfies the following equation:

$$
\begin{gathered}
\partial_{t} w=v \Delta w-u \nabla u-\nabla p \\
\nabla \cdot w=0
\end{gathered}
$$

As the heat equation preserves the divergence condition, the following equation is obtained $\nabla \cdot w=0$ for all $t \geq 0$. Setting:

$$
\begin{gathered}
\gamma_{r}=\left\|A^{r} w\right\|_{L^{2}}^{2} \\
\zeta_{r}=\left\|A^{r} \mathrm{e}^{\tau A} w\right\|_{L^{2}}^{2}
\end{gathered}
$$


And repeating the steps, the following result is obtained:

$$
\begin{aligned}
& \frac{1}{2} \dot{\zeta}_{r} \leq i \zeta_{r}^{1 / 2} \zeta_{r+1}^{1 / 2}-v \zeta_{r+1}+c_{1}\left(G_{s}^{1 / 2}+G_{r}^{1 / 2}\right) G_{r+1}^{1 / 2} G_{r}^{1 / 2}+c_{1}\left(G_{s+1}^{1 / 2}+G_{r+1}^{1 / 2}\right) G_{r} \\
& =\left(\frac{1}{2} \frac{\dot{\tau}}{\tau}-\frac{v}{8} \frac{1}{\tau^{2}}\right) \zeta_{r}+\left(\frac{1}{2} \tau \dot{\tau}-\frac{v}{8}\right) \zeta_{r+1}-\frac{v}{16} \frac{1}{\tau^{2}} \zeta_{r}+\frac{c_{3}}{2} \frac{v}{\tau^{2(r+1)}} \zeta_{0}+O\left(\frac{1}{\tau^{3 \gamma+5 r / 2+1}}\right)
\end{aligned}
$$

The second of nonlinear terms arises from (47) by using and choosing the smallest possible $s=r / 2$. For $r \leq 2$, the following is obtained:

$$
\begin{aligned}
\left(G_{s+1}^{1 / 2}+G_{r+1}^{1 / 2}\right) G_{r} & =\left(G_{r / 2+1}^{1 / 2}+G_{r+1}^{1 / 2}\right) G_{r}=G_{r / 2+1}^{1 / 2} G_{r}+\text { higher_order_terms } \\
& =O\left(\frac{1}{\tau^{\gamma+r / 2+1}}\right) O\left(\frac{1}{\tau^{2(\gamma+r)}}\right)+\text { higher_order_terms }
\end{aligned}
$$

The following differential inequality is obtained:

$$
\dot{\zeta}_{r}(t) \leq-\frac{\delta}{\tau^{2}} \zeta_{r}+\frac{\varepsilon c_{8}}{\tau^{2(\gamma+r+1)}}+O\left(\frac{1}{\tau^{3 \gamma+5 r / 2+1}}\right)
$$

And the following is obtained:

$$
\left\|A^{m} w\right\|^{2} \leq \frac{\varepsilon c_{11}(m, r)}{\tau^{2(\gamma+m)}}+\text { higher_order_terms }
$$

After having proved that solution for $\tau$ exists and if we examine the equation, as $\tau \rightarrow \infty$ the distance between heat equation solution and Navier-Stokes equation demonstrates convergence and if the following heat equation solution is found then the solution for Navier-Stokes equations exist and is in the same range as heat equation solution.

Now the heat solution equation Cannon [4] is analyzed. The solution of heat equation:

$$
\left(\partial_{t}-\Delta\right) u=0
$$

Satisfies a mean-value property

$$
\Delta u=0
$$

Precisely if $u$ solves

$$
\left(\partial_{t}-\Delta\right) u=0
$$

And

$$
(x, t)+E_{\lambda} \subset \operatorname{dom}(u)
$$

Then

$$
u(x, t)=\frac{\lambda}{4} \int_{E_{\lambda}} u(x-y, t-s) \frac{|y|^{2}}{s^{2}} \mathrm{~d} s \mathrm{~d} y
$$

where $E_{\lambda}$ is a heat ball,

$$
\begin{gathered}
E_{\lambda}:=\{(y, s): \Phi(y, s)>\lambda\}, \\
\Phi(x, t):=(4 t \pi)^{-\frac{n}{2}} \exp \left(-\frac{|x|^{2}}{4 t}\right) .
\end{gathered}
$$

Notice that

$$
\operatorname{diam}\left(E_{\lambda}\right)=o(1)
$$


So that $\lambda \rightarrow \infty$ demonstrates that equation is existent and is captured in the ball if the $\lambda$ is finite.

The previous assumptions and results prove the existence of smooth and strong Navier-Stokes solution of equation in $R^{3}$ and represent the solution of millennium problem in $R^{3}$.

\section{Conclusion}

It is proved that the strong solution of Navier-Stokes equation is smooth, existent and unique. Firstly, turbulent solutions are defined and it is proved that they are strong solution, but as the turbulent solutions are only possible for small time intervals, it is tried to extend the time interval by using the Equation (39) and it is proved that the differential inequality (40) holds at the same time for some $\tau$ that does not converge to 0 . Then the result is established, it is demonstrated that solutions exhibit possible finite blow-up time, which means that they exist and persist in the system. In order to establish if the solution exists for the finite time, the heat equation solution and Navier-Stokes solution are compared. It is proved that two solutions converge as $\tau \rightarrow \infty$ which proves the existence of solution in infinite time. If a surgery procedure is applied, the solution exists for some time, then blows up, then arises again and that process repeats. This statement proves that the solution is either existent or periodic, but it exists all the time. It is possible to introduce a stochastic process in order to explain the existence of the dynamical periodic solution, but this is left for further research. This paper proves the existence of Navier-Stokes solution in $R^{3}$ and represents a breakthrough in fluid dynamics analysis.

\section{Acknowledgements}

I would like to thank my family, my favourite aunt, my grandparents who had a tremendous influence on my love towards mathematics. I want to thank my aunt Cica, my aunt Sonja and her husband Voja, her daughters, my uncle Nemanja and his family and all other relatives who provided me immense support. Love you all.

\section{References}

[1] Oliver, M. and Titi, E.S. (2000) Remark on the Rate of Decay of Higher Order Derivatives for Solutions to the NavierStokes Equations in $\mathrm{R}^{n}$. Journal of Functional Analysis, 172, 1-18. http://dx.doi.org/10.1006/jfan.1999.3550

[2] Okabe, T. (2009) Asymptotic Energy Concentration in the Phase Space of the Weak Solutions to the Navier-Stokes Equations. Journal of Differential Equations, 246, 895-908. http://dx.doi.org/10.1016/j.jde.2008.07.037

[3] Tao, T. (2006) Perelman’s Proof of the Poincaré Conjecture: A Nonlinear PDE Perspective. http://arxiv.org/abs/math/0610903

[4] Cannon, J.R. (1984) The One-Dimensional Heat Equation. Vol. 23. Cambridge University Press, Cambridge. http://dx.doi.org/10.1017/CBO9781139086967 